Rev. Adm. Saúde Vol. 17, Nº6, Jan. - Mar. 2017

ARTIGO ORIGINAL

\title{
O Uso do KAN BAN na Gestão do Cuidado: Superando Limites.
}

El Uso de Kanban en la Gestión de la Atención: la Superación de los Límites.

The Use of Kanban in Care Management: Overcoming Limits.

Isis Aparecida Cunácia Massaro ${ }^{1}$, Altair Massaro²

1. Médica nefrologista, especialista em Cuidados Paliativos. Docente colaboradora do Instituto de Ensino e Pesquisa do Hospital Sírio-Libanês de São Paulo e docente da Medicina da Universidade São Caetano do Sul.

2. Médico, mestre em Saúde Coletiva. Docente colaborador do Instituto de Ensino e Pesquisa do Hospital Sírio-Libanês de São Paulo e docente da Medicina da Universidade São Caetano do Sul.

\section{RESUMO}

Considerando o tempo de permanência hospitalar como indicador da qualidade assistencial e elemento fundamental para o enfrentamento da superlotação dos hospitais, a proposta deste artigo é promover o entendimento do uso do KAN BAN, como ferramenta a ser utilizada para otimizar o tempo de permanência e contribuir para a melhora no fluxo do paciente no âmbito hospitalar.

Palavras-chave: Tempo de espera. KAN BAN. Tempo de Permanência. Governança Clínica e Gestão da Qualidade.

\section{RESUMEN}

Teniendo en cuenta la duración de la permanencia hospitalaria como un indicador de una atención de calidad y un elemento clave para combatir el hacinamiento de los hospitales, el propósito de este artículo es promover la comprensión de la utilización de KAN BAN, como una herramienta que se 
utiliza para optimizar la duración de la estancia y contribuir a la mejora en el paciente fluya en los hospitales.

Palabras clave: Tiempo de Permanencia. Gestión Clínica e Gestión de la Calidad.

\section{ABSTRACT}

Considering the length of hospital stay as an indicator of healthcare quality and fundamental group unit to combat the overcrowding of hospitals, the purpose of this paper is to promote the understanding of the use of KAN BAN, as a tool to be used to optimize the length of stay and contribute to the improvement in patient flow in hospitals

Keywords: Waiting-time. Length of Stay. Clinical Governance.Lean principles. Emergency services.

\section{INTRODUÇÃO}

Um dos maiores desafios para os sistemas de saúde é adequar a sua capacidade de oferta em face às necessidades em saúde das populações e dos indivíduos ${ }^{1}$.

Em geral, os serviços de saúde no Brasil são bastante precarizados, onde boa parte sofre dificuldades estruturais, tanto em relação aos espaços físicos como em seu parque tecnológico e também na alocação e capacitação de seus profissionais. Além disso, há pouco processo de planejamento das ações e políticas de avaliação de resultado. Esta situação dificulta muito os processos de trabalho tanto assistenciais como de gestão.

Nos serviços de atenção às emergências, especialmente nos grandes hospitais brasileiros, esta situação tem como reflexo imediato a superlotação de suas portas de entrada.

\section{O PROBLEMA}

"A superlotação nos Serviços de Emergência Hospitalar (SEH) é um fenômeno mundial, caracteriza-se por: todos os leitos do SEH ocupados; pacientes acamados nos corredores; tempo de espera para atendimento acima de uma hora; alta tensão na equipe assistencial; grande pressão para novos atendimentos. Indica, em última instância, baixo desempenho do sistema de saúde, como um todo e do hospital em particular, e induz à baixa 
qualidade assistencial.", E ainda, pode representar uma ameaça aos direitos humanos tanto dos usuários quanto dos trabalhadores. ${ }^{3}$

As causas da superlotação são multifatoriais e amplamente citadas na literatura. ${ }^{4,5}$ Sabemos que, com frequência, os serviços de urgências e emergências são utilizados como uma porta de acesso à rede de saúde. Aliado a isso, temos um aumento da população demográfica e envelhecimento de parcela considerável desta. Este fato encontra-se diretamente associado ao incremento da prevalência de doenças crônicas e agudizações da mesma.

A superlotação é percebida por todos visualmente e tem como seu principal marcador o aumento do tempo de permanência. ${ }^{6}$

\section{A BUSCA DAS SOLUÇÕES}

As tentativas de modificar essa realidade e melhorar o fluxo dos pacientes e reduzir o seu tempo de permanência, são avaliadas e apresentadas em vários estudos. ${ }^{7,8}$ Diante do exposto, se faz necessário o uso de ferramentas para a redução do tempo de permanência, observando a relação tempo e risco e as necessidades dos pacientes com a intenção de melhorar o cuidado prestado. Nesse sentido de buscar ferramentas que possam auxiliar a minorar a superlotação com olhar para o risco, os serviços de urgências e emergências tiveram avanços ao implantar a tecnologia do Acolhimento com Classificação de Risco (ACCR). Segundo Heisler, a ACCR ainda não é suficiente para monitorar o tempo de permanência e o gerenciamento de fluxo não é garantido apenas pela classificação inicial dos pacientes. Nesse ponto devemos lançar mão do uso da ferramenta KAN BAN.$^{9} \mathrm{E}$ continuando a citar o último autor:

"O sistema KAN BAN nas emergências hospitalares pode ser entendido como uma ferramenta de qualificação do gerenciamento do cuidado, por sítios assistenciais. Dessa forma, através dessa ferramenta podemos identificar o paciente, identificar a equipe responsável, localizar o paciente na emergência, indicar o tempo de permanência, entre outros."

KAN BAN, formado por dois Kanji, que são caracteres da língua japonesa adquiridos a partir de caracteres chineses, possui vários significados: cartão, símbolo (cores de identificação) ou painel. Em linhas gerais é um sistema de controle da produção, cujo objetivo é minimizar os estoques de material em processo, produzindo em pequenos lote, somente o necessário e no tempo certo. $^{10}$

KAN BAN e Just in Time são conceitos da área de produção e não raras vezes, são confundidos quando aplicados a outros campos de conhecimento. O Sistema KAN BAN foi desenvolvido a partir do conceito simples de aplicação da gestão visual no controle de produção e estoques com a função primordial de viabilizar a produção "Just inTime"11. Portanto o ganho real no sistema produtivo advém do funcionamento da operação - essa 
compreensão é fundamental - e não necessariamente da aplicação ou não da gestão visual. ${ }^{10}$

Ao transportamos essa ferramenta para a saúde, o principal ganho reside no fato de que ao diagnosticarmos o tempo de permanência que o paciente encontra-se em uma determinada unidade, tem o potencial, através da visualização, de disparar a necessidade de identificar a(s) causa(s) que determinam a demora e intervir sobre a(s) mesma(s). E consequentemente isso impactará na melhor assistência prestada.

Operacionalmente, existem dois modos de utilização do KAN BAN: placa e planilha. Estes dois modos se complementam e, portanto, é necessário que seu uso ocorra paralelamente.

O uso das placas é feito à beira do leito. Para tanto, o primeiro passo é a definição dos níveis de permanência para cada coorte de usuários a ser considerado. Por exemplo, se a coorte é uma enfermaria onde usualmente são internados pacientes clínicos, cujas patologias prevalentes, têm expectativa de recuperação, baseando-se em evidencias na literatura, de 7 dias, os níveis de aceitabilidade podem ser: Nível 1 (KAN BAN I ou Verde dentro dos limites do esperado) de até 7 dias, nível 2 (KAN BAN II ou Amarelo - sinal de alerta) entre 7 e 9 dias e nível 3 (KAN BAN III ou Vermelho - sinal de intervenção) acima de 10 dias. Se a coorte é uma unidade de emergência, por exemplo, a sala amarela, os níveis de aceitabilidade de tempo devem ser diferentes. Sabe-se que a permanência nas unidades de urgência e emergência deve ser a menor possível, assim, limites como 24 horas para o Nível 1 (KAN BAN I ou Verde), entre 25 e 36 horas para nível 2 (KAN BAN II ou Amarelo) e acima de 37 horas para nível 3 (KAN BAN III ou Vermelho) são bastante pertinentes. De modo que, cada limite deve ser pactuado com as equipes tendo como base a série histórica local, mas buscando evidências cientificas que servirão de parâmetros a serem atingidos. Estas equipes monitoram e diagnosticam as causas do aumento da permanência e as solucionam. Este, portanto, é o segundo passo, ou seja, a identificação das causas que aumentam a permanência dos pacientes e tornam o KAN BAN vermelho ou III e a solução conjunta destas situações. O padrão ouro é atuar com antecedência ao aumento da permanência, ou seja, ainda no KAN BAN I (verde) ou II (amarelo).

Assim, a constituição de equipes de referência para o cuidado aos pacientes deve levar em conta o trabalho interdisciplinar, ou seja, sempre promovendo a troca de saberes entre os profissionais e buscando soluções mais potente para cada problema identificado ${ }^{12}$. O processo de trabalho, deste modo, consiste em visitas diárias a cada paciente pela equipe de referência, onde se poderá refletir conjuntamente, além das questões clínicas pertinentes ao cuidado, o status atualizado do KAN BAN e as suas causas. Estas motivações, evidentemente, podem pertencer ao campo clínico, ou seja, a permanência está acima do esperado por complicações inerentes ao processo de adoecimento singular de cada um; todavia, mesmo nesta situação ainda cabe a reflexão da equipe sobre a adequação do tratamento proposto e sua condução, de forma a ajustar sempre o projeto terapêutico. Entretanto, outras causas também são bastante incidentes, como: demora na 
realização e/ou resposta de exames subsidiários, falta de insumos necessários ao tratamento, demora na avaliação por especialistas (equipe matricial), necessidades sociais etc. Todas estas causas singulares devem ser tratadas pela equipe de referência, procurando soluções potentes e rápidas, no sentido de reduzir a permanência desnecessária do usuário no ambiente hospitalar.

Algumas situações ainda exigem um pouco de reflexão. A primeira se deve à continuidade do cuidado em diversas unidades do hospital, por exemplo, o paciente que está na sala verde de um pronto socorro e depois é internado na enfermaria do mesmo serviço; a questão decorrente é se devemos manter a contagem do KAN BAN iniciado no pronto socorro na nova unidade ou se começaria nova contagem ao ingressar na enfermaria?

A resposta exige que pensemos como as equipes estão aptas e apropriadas a lidar com a ferramenta. A melhor hipótese é que tenhamos em mente o tempo total de permanência hospitalar, desde a sua estada no pronto socorro e a complementação na enfermaria e também, um segundo KAN BAN, que aponte apenas a nova permanência. Isso, todavia, pode ser dificultador do processo de trabalho de equipes ainda neófitas à tecnologia; neste caso, apenas o KAN BAN da nova unidade deveria ser sustentado, mas sempre levando em conta o tempo total de internação do paciente para construção de seu projeto terapêutico; deste modo, o KAN BAN da enfermaria refletirá o processo de trabalho e o plano de cuidado adotado na unidade e suas possíveis necessidades de ajustes.

O segundo aspecto se deve à seleção de coortes para definição dos parâmetros de tempo (níveis) do KAN BAN. Algumas unidades assistenciais têm prevalências muito distintas e gostariam de usar KAN BAN diferentes para distintos grupos, por exemplo, em uma enfermaria pacientes com uma dada patologia podem ter expectativas de recuperação e, portanto, de permanência esperada diferente de outras. A reflexão é a mesma que a anterior, ou seja, se a equipe tem habilidade para lidar com a ferramenta KAN BAN e destreza em seu manuseio, produzindo discussões potentes e ágeis, nada impede que possa implementar níveis distintos para subgrupos no mesmo espaço, tudo depende da pactuação e forma de lidar da equipe. Todavia, se a equipe ainda carece de experimentação e prática, utilizar um parâmetro único para a mesma unidade pode facilitar o processo de trabalho e acompanhamento dos casos. Nesta hipótese, é fundamental a pactuação dos parâmetros pela equipe, respeitando um corte médio entre as expectativas de permanência dos distintos pacientes, sempre fundamentados na série histórica local e na evidência da literatura especializada.

O segundo modo de utilização do KAN BAN são as planilhas. Este instrumento pode ser elaborado, por exemplo, em ferramenta de informática do tipo Excel ou similar, em softwares específicos ou mesmo manualmente.

Estas planilhas nada mais são que o consolidado das informações das placas na beira do leito, num dado período em uma certa unidade. Os dados registrados podem ser: nome do paciente, idade, data da internação no hospital e na unidade, necessidades em saúde identificadas pela equipe e, 
fundamentalmente, nível do KAN BAN e causa para KAN BAN III (Vermelho), outros dados que a equipe julgue relevantes podem ser acrescidos a esta lista.

A alimentação da planilha é responsabilidade da equipe de referência de cada usuário. Este instrumento, uma vez atualizado, pode ser mantido apenas no computador - quando em meio eletrônico - ou exposto à equipe em um painel (meio físico) ou uma tela (meio eletrônico), neste caso, tomando-se os cuidados com a preservação do sigilo ético necessário.

Se o uso do KAN BAN na sua forma de placa tinha o foco da equipe de referência, o uso das planilhas de consolidados devem ser, além de objeto de acompanhamento das mesmas equipes, acompanhadas pelo colegiado gestor da unidade ou outros grupos gestores como por exemplo o Núcleo de Acesso a Qualidade Hospitalar (NAQH).

O foco de análise para os profissionais que integram os colegiados de gestão está no aspecto coletivo, ou seja, as informações extraídas do consolidado de dados em um dado período em uma certa unidade. Deste modo, dois indicadores podem ser observados que ajudarão o processo de gestão da unidade: o primeiro é definido como Taxa de Saídos no KAN BAN III (Vermelho), este indicador refere-se a porcentagem de pacientes que saíram no KAN BAN III num dado período. Para efeito de reflexão, tomemos o seguinte exemplo: numa dada enfermaria no mês de abril a Taxa de Saídos no KAN BAN III foi de $20 \%$; dois meses depois este mesmo indicador nos mostra uma ascensão para $40 \%$; isso, sem sombra de dúvidas, deve ser um fator bastante mobilizador da equipe, cuja pergunta, na sequência, deverá ser: o que ocorreu neste período? Isto nos leva ao segundo indicador: as causas mais prevalentes relacionadas ao aumento da permanência. Estas informações, portanto, trarão elementos bastante substanciais para gestão de processos de trabalho e arranjos assistências que produzirão adequação à média de permanência na unidade e melhora da qualidade assistencial. Deste modo, estes indicadores devem fazer parte do monitoramento da qualidade da assistência da unidade.

\section{CONCLUSÕES}

É muito importante entendermos que ferramenta KAN BAN tem o potencial de instigar essas intervenções, mas não por si só fará com que aconteçam. Com isso queremos marcar que o seu uso somente será eficaz quando manejado por uma equipe de referência do cuidado e para a qual o emprego do KAN BAN faça sentido. Caso contrário será simplesmente uma sinalização visual, cuja essência seria meramente burocrática e sem consistência para disparar todos os processos. Encontramos consonância com esse pensamento, no Estudo de Lemieux-Charles et al. que diz que existe uma necessidade de comprometer o profissional com as mudanças organizacionais, sociais e culturais, mais do que procurar um "tiro mágico" (magic bullet) para as mudanças do comportamento individual13. 


\section{REFERÊNCIAS}

1. FEUERWEKER, L.M. Modelos tecnoassistenciais, gestão e organização do trabalho em saúde: nada é indiferente no processo de luta para a consolidação do SUS. Interface (Botucatu), v.9, n.18, p.489-506, set/dez 2005.

2. COMMITTEE ON THE FUTURE OF EMERGENCY CARE IN THE UNITED STATES HEALTH SYSTEM. Hospital-based emergency care: at the breaking point. Washington; DC: National Academies Press; 2007.

3. REGNER, A.P.; NASCIMENTO, R.I.M. Enfrentamento da superlotação da emergência do HNSC: oportunizando a discussão sistêmica do modelo de atenção do HNSC. Gerência de pacientes externos/Grupo Hospitalar Conceição. [Relatório Social] Porto Alegre (RS): 2011.

4. WOMACK, J.P. Going lean in healthcare. Cambridge, MA: Institute for Healthcare Improvement, 2005.

5. CONDELL, J.L.; SHARBAUGH, D.T.; RAAB, S.S. Error-free pathology: applying Lean production methods to anatomic pathology. Clinical Laboratory of Medicine, v.24, p.865-99, 2004.

6. BRADLEY V.M. Placing Emergency Department crowding on the decision agenda. Journal of Emergency Nursing, v.31, p.247-58, 2005.

7. Institute of Medicine. To err is human: building a safer health system. Washington, DC: National Academy Press, 1999.

8. PERSOON T.J.; ZALESKI S.; FRERICHS J. Improving preanalytic processes using the principles of Lean production (Toyota Production System). Am J Clin Pathol.125:16-25, 2006.

9. HEISLER P.A. Aplicação da metodologia KAN BAN como ferramenta adaptada para gestão de "leitos" na emergência [Trabalho de Conclusão de Curso]. Porto Alegre, RS: Fundação Oswaldo Cruz, Instituto de Comunicação e Informação Cientifica e Tecnológica em Saúde, 2012.

10. MOURA, R.A. A simplicidade do controle da produção. São Paulo: Instituto de Movimentação, 1989.

11. OHNO, T. O sistema Toyota de produção: além da produção em larga escala. Porto Alegre, RS: Bookman, 1997.

12. ALMEIDA, N.F. Transdisciplinaridade e o paradigma pós-disciplinar na saúde. Saúde e Sociedade, v.14, n.3, p.30-50, 2005.

13. LEMIUEX-CHARLES, L.; CHAMPAGNE, F. Introduction: towards a broader understanding of the use of knowledge and evidence in health care. In: Lemiuex-Charles L, Champagne F, editors. Using knowledge 
and evidence in health care: multidisciplinary perspectives. Toronto:

University of Toronto Press; p.317, 2004.

14.BITTENCOURT, R.J.; HORTALE, V. Intervenções para solucionar a superlotação nos serviços de emergência hospitalar: uma revisão sistemática. Cadernos de Saúde Pública, Rio de Janeiro, RJ, v. 25, n. 7, 1439-54, 2009.

Recebido: 13/01/2017; Publicado: 17/01/2017

Correspondência: Altair Massaro. E-mail: altair.massaro@gmail.com

Conflito de Interesses: os autores não declararam conflito de interesses

(C) This is an Open Access article distributed under the terms of the Creative Commons Attribution License, which permits unrestricted use, distribution, and reproduction in any medium, provided the original work is properly cited. 